\title{
MicroRNA Expression Profiling in PBMCs: A Potential Diagnostic Biomarker of Chronic Hepatitis C
}

\author{
Chiu-Chun Chang, ${ }^{1,2}$ Chun-Che Lin,, ${ }^{2,3}$ Wan-Ling Hsieh, ${ }^{2,4}$ Hsin-Wu Lai, ${ }^{2}$ \\ Chia-Hsun Tsai, ${ }^{2}$ and Ya-Wen Cheng ${ }^{5}$ \\ ${ }^{1}$ Institute of Medicine, Chung Shan Medical University, Taichung, Taiwan \\ ${ }^{2}$ Division of Gastroenterology, Department of Internal Medicine, Chung Shan Medical University Hospital, Taichung, Taiwan \\ ${ }^{3}$ School of Medicine, Chung Shan Medical University, Taichung, Taiwan \\ ${ }^{4}$ Department of Pathology, Chung Shan Medical University, Taichung, Taiwan \\ ${ }^{5}$ Graduate Institute of Cancer Biology and Drug Discovery, College of Medical Science and Technology, Taipei Medical University, \\ Taipei 115, Taiwan
}

Correspondence should be addressed to Ya-Wen Cheng; ywc@tmu.edu.tw

Received 1 July 2014; Revised 11 September 2014; Accepted 27 September 2014; Published 18 November 2014

Academic Editor: Vincent Sapin

Copyright (C) 2014 Chiu-Chun Chang et al. This is an open access article distributed under the Creative Commons Attribution License, which permits unrestricted use, distribution, and reproduction in any medium, provided the original work is properly cited.

\begin{abstract}
The expression levels of miR-16, miR-193b, miR-199a, miR-222, and miR-324 in PBMCs were significantly higher in CHC patients compared with healthy controls and significantly different between CHC patients with HCV genotype 1 (GT-1) and non-genotype-1 (non-GT-1). Multivariate logistic regression analysis also showed that patients with high expression levels of the six target miRNAs had an approximately 7.202-fold risk of CHC compared with those with low expression levels of the target miRNAs. We concluded that the expression levels of miR-16, miR-193b, miR-199a, miR-222, and miR-324 target miRNAs in PBMCs of CHC may act as significant risk biomarkers for the development of CHC.
\end{abstract}

\section{Introduction}

Hepatitis C virus (HCV) infection affects more than $3 \%$ of the world's population [1]. HCV infection frequently induces chronic liver disease, ranging from chronic hepatitis $\mathrm{C}(\mathrm{CHC})$ to liver cirrhosis and hepatocellular carcinoma (HCC) [1]. HCV can interact with host cells to modulate cell survival signaling, alter gene expression, and induce cell transformation [2].

miRNAs are endogenous, small (approximately 22nucleotide) noncoding RNAs that downregulate gene expression [3]. Some miRNAs are produced at high concentrations within cells in a tissue-specific manner $[4,5]$, and such miRNAs have recently been reported to be remarkably stable in plasma $[4,6]$. miRNA-122 (miR-122) is a highly abundant, liver-expressed microRNA [7, 8]. Research has shown that miR-199a directly regulates HCV replication and suggested that it may serve as a novel antiviral therapy [9]. In addition, a previous computational study of HCV signaling pathways suggested a critical role for miRNAs in the replication, propagation, and latency of viruses in the host cell [10]. Specifically, the study revealed that miR-122, miR-320, and miR-191 were downregulated and that miR-215, miR-16, miR26, miR-130, miR-199, and miR-155 were upregulated in HCV infected cells [11]. Based on these, findings suggest that miRNAs have the potential to become novel drug targets in viral-induced infectious or malignant diseases [11].

An ideal biomarker of $\mathrm{CHC}$ should be released into the systemic circulation or other body fluids, where it can be detected in a blood-based assay or assay of another accessible body fluid [12-14]. Peripheral blood mononuclear cells (PBMCs) have been reported to play an important role in HCV progression [15]. Induction of PBMC-miR-155 was found in patients with chronic HCV infection [16]. In addition, HCV core induces STAT3 as a result of the alteration of inflammatory response by antigen-presenting cells via 
TABLE 1: The clinical information of CHC patients in this study.

\begin{tabular}{lc}
\hline Parameters & \\
\hline Gender, male/female & $60 / 31(2: 1)$ \\
Age (years) & $55.1 \pm 10.6$ \\
BMI & $24.5 \pm 3.4$ \\
HCV RNA $(\log I U / m L)$ & $6.28 \pm 0.93$ \\
AST & $82.7 \pm 75.5$ \\
ALT & $121.3 \pm 97.9$ \\
$\gamma$ GT & $88.6 \pm 134.1$ \\
Cr & $0.79 \pm 0.19$ \\
Cholesterol & $163.1 \pm 30.1$ \\
TG & $136.0 \pm 147.8$ \\
Glucose & $123.7 \pm 47.4$ \\
WBC & $5878.8 \pm 1580.8$ \\
Hb & $14.9 \pm 1.5$ \\
PLT: $>15000 /<15000$ & $53 / 38$ \\
Genotype-1/non- 1 & $71 / 20$ \\
\hline
\end{tabular}

CHC: chronic hepatitis C; BMI: body mass index; AST: aspartate aminotransferase; ALT: alanine aminotransferase; Cr: creatinine; TG: triglyceride; PLT: platelet.

an IL-6 autocrine pathway [17]. There are several diseaseassociated miRNAs in PBMC reported in previous studies $[18,19]$. Thus, expression levels of miRNAs in PBMC may correlate to $\mathrm{HCV}$ infection and treatment outcome to anti$\mathrm{HCV}$ therapy and potentially serve as noninvasive markers.

In this study, we analyzed the expression profiles of miRNAs in PBMCs of CHC patients and healthy controls. Specific expression patterns found in the $\mathrm{CHC}$ patients relative to the healthy controls suggested that miRNA activities may potentially contribute to the pathobiology of $\mathrm{HCV}$ infection and could be useful diagnostic biomarkers.

\section{Material and Methods}

2.1. Study Population. Peripheral blood samples were collected from $91 \mathrm{CHC}$ patients who were treated with IFN alpha plus ribavirin at the Department of Internal Medicine, Chung Shan Medical University Hospital, and from 48 healthy controls, between October 2010 and December 2012. Informed written consent was obtained from all of the subjects and/or guardians prior to the use of their blood specimens. The acquisition of the samples and their subsequent examination were approved by the Institutional Review Board of Chung Shan Medical University. None of the participants had a previous history of cancer. The demographic and clinical data of the patients at the time of the sample collection are summarized in Table 1.

All participants gave their informed consent, and the following criteria were met. (i) All patients had an established diagnosis of CHC. (ii) All patients had an absence of another cause of chronic liver disease and an absence of viral coinfection. (iii) All patients received treatment with either PEG-IFN- $\alpha 2$ b (Viraferonpeg; Schering Plough) at a dose of $1.5 \mu \mathrm{g} / \mathrm{kg}$ of body weight/week and weight-based ribavirin at a dose of 800 to $1,200 \mathrm{mg} /$ day (Rebetol; Schering Plough) or PEG-IFN- $\alpha 2 \mathrm{a}$ at a dose of $180 \mu \mathrm{g} /$ week (Pegasys; Roche) and weight-based ribavirin at 1,000 to $1,200 \mathrm{mg} /$ day (Copegus;
Roche). The duration of treatment was 48 weeks for the patients with HCV genotypes 1 and 4 and 24 weeks for those with genotypes 2 and 3. For patients with HCV genotypes 1 and 4, if the HCV RNA was detectable at week 24, the treatment was stopped. For those with HCV genotype 1, if the HCV RNA decreased less than 2 log10 from the baseline to week 12 of therapy, treatment was stopped. (iv) All individuals with CHC enrolled in this study had known HCV genotypes. (v) Blood samples were collected before and after IFN alpha plus ribavirin treatment. Tumor tissues were collected from hepatocellular carcinoma patients infected with HCV to be used to compare the miR-122 expression.

2.2. MicroRNA Profiling. Low-molecular weight RNAs of less than $200 \mathrm{nt}$ were extracted from the PBMCs of 10 $\mathrm{CHC}$ patients and a reference pool of 10 paired healthy controls. The total RNA was extracted from the PBMCs of the HCV patients using TRIzol (Invitrogen, Carlsbad, CA), where the TRIzol was supplemented with $0.2 \mathrm{~mL}$ of chloroform per $1 \mathrm{~mL}$ of reagent, mixed, and centrifuged (15 min; $\left.12,000 \mathrm{~g} ; 4^{\circ} \mathrm{C}\right)$. The supernatant was retained. To pellet the RNA, $0.5 \mathrm{~mL}$ of isopropanol (per $1 \mathrm{~mL}$ of reagent) was added and the mixture was incubated for $10 \mathrm{~min}$ at room temperature, followed by centrifugation $(15 \mathrm{~min}$; $\left.12,000 \mathrm{~g} ; 4^{\circ} \mathrm{C}\right)$. The pellet was washed once in $0.5 \mathrm{~mL}$ of $75 \%$ ethanol. The final RNA products were quantified by absorbance measurements at $260 \mathrm{~nm}$ (A260) and $280 \mathrm{~nm}$ (A280). The A260/A280 values were higher than 1.6 for all of the samples. A measure of $5 \mu \mathrm{L}$ of RNA was reversetranscribed using the TaqMan miRNA reverse-transcription kit. The miRNA profiles were analyzed by MegaplexTM Pools for MicroRNA Expression Assays (MegaplexTM RT Primers, Human Pools A and B; Applied Biosystems, Foster City, CA). The detailed microRNA list is shown on the following website: http://www.lifetechnologies.com/order/catalog/product/4444745/.

2.3. Real-Time PCR-Based Detection of MicroRNAs. The detailed methods of isolating short RNAs and the reversetranscription reaction were the same as those described in Section 2.2. The expression of mature miRNA was detected by a TaqMan miRNA assay according to the instructions of the manufacturer (Applied Biosystems) and normalized using the $2-\Delta \Delta$ CT-method relative to U6-snRNA. The CT (cycle threshold) was inversely proportional to the amount of target nucleic acid in the sample, and all of the TaqMan-PCRs were performed in triplicate. The definitions of the high and low expressions of the microRNA were dependent on the mean value of all patients. The expression levels higher than the mean were defined as high expressions, while the expression levels lower than the mean were defined as low expressions.

2.4. Statistical Analysis. All data were analyzed using the Statistical Package for the Social Sciences version 13.0 software (SPSS Inc., Chicago, IL). The Chi-square test $\left(\chi^{2}\right.$ test) was used to compare the expression of miRNAs and clinicopathological parameters. A probability of less than 0.05 was considered statistically significant. To determine 


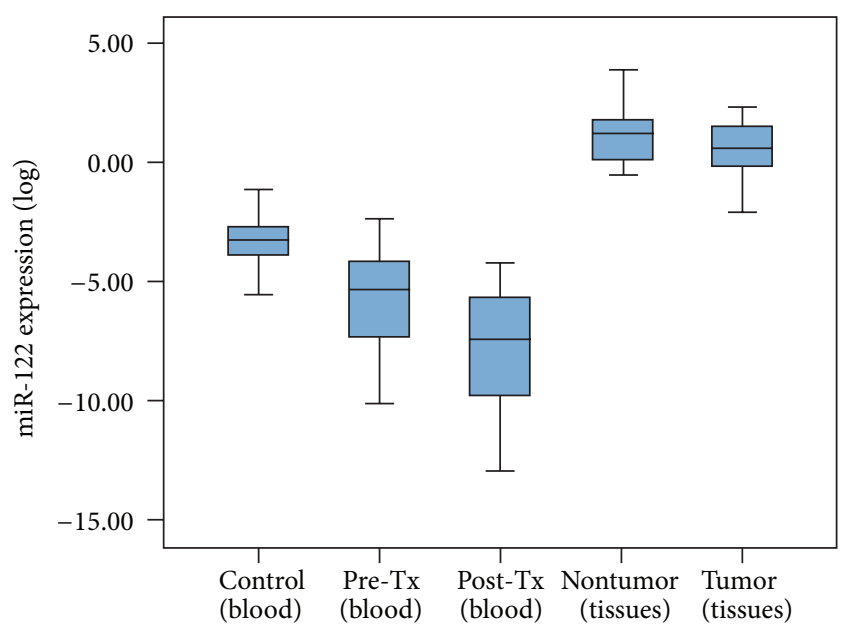

FIgUre 1: The expression levels of miR-122 in PMBCs of $\mathrm{CHC}$ patients and healthy controls were significantly lower than in tumor and nontumor tissues of liver cancer. The miR-122 expression level was detected by real-time PCR.

whether the expression of the miRNAs could be used as an independent risk factor for HCV infection, a multiple unconditional logistic model was used to obtain the adjusted odds ratios (ORs) for the difference in gender, age, and BMI, and a corresponding 95\% CI was used for the T-score after adjusting for the effect of potential confounding factors. The factors used in the multivariate analysis were analyzed by univariate analysis first. If the $P$ value was less than 0.05 , they were subjected to multivariate analysis.

\section{Results}

3.1. miR-122 Was Downregulated in the PBMCs of CHC Patients. Previous reports showed that the expression levels of miR-122 were decreased in the liver tissues of $\mathrm{CHC}$ patients $[7,8,11,20]$. To understand whether the expression levels of miRNAs in PBMCs could be used as biomarkers for $\mathrm{CHC}$ diagnosis, the expression of miR-122 was used as a control. As shown in Figure 1, the expression levels of miR-122 in the PBMCs of the CHC patients were significantly lower than in the healthy controls $(P<0.001)$. We used 20 paired tumor and nontumor HCC tissues to confirm the results from the PBMCs of the CHC patients (Figure 1). The expression of miR-122 was higher in the normal tissues than in the tumor tissues of the $\mathrm{CHC}$ patients. The results are consistent with previous reports $[8,9,12,16]$. We also found that the expression levels of miR-122 in the tumor and the normal tissues of the HCC patients were significantly higher than in the PBMCs of the CHC patients and that the expression of miR-122 in the PBMCs of the CHC patients was significantly lower than in the healthy controls.

3.2. Expression Levels of MicroRNAs Were Associated with HCV Genotypes and Differed between the CHC Group and the Healthy Controls. We analyzed miRNA expression profiles in the PBMCs of $10 \mathrm{CHC}$ patients and 10 paired healthy controls using a miRNAs array. We identified miRNAs that were significantly differentially expressed in the $\mathrm{CHC}$ patients and the control group. The results of a global miRNA expression analysis of the PBMCs of the $91 \mathrm{CHC}$ patients compared with the 48 healthy donors are shown in Table 2. We selected five target miRNAs (miR-199a-3p, miR-324, miR-16, miR-222, and miR-214) that had been studied in HCC tumor tissues for further analysis. To determine whether the expression levels of the miRNAs could be used as diagnostic markers of $\mathrm{CHC}$, their expression levels in the PBMCs of the $\mathrm{CHC}$ patients and the healthy controls were analyzed by realtime RT-PCR. As shown in Table 3, the expression levels of miR-122, miR-16, miR-193b, miR-199a, miR-222, and miR-324 were significantly higher in the PBMCs of the CHC patients compared with the healthy controls (miR-16, $P=0.001$; miR-122, $P<0.001$; miR-193b, $P<0.001$; miR-199a, $P<$ 0.001 ; miR-222, $P<0.001$; and miR-324, $P=0.001$ ). No significant difference was found in the expression of miR-214 between the $\mathrm{CHC}$ group and the healthy controls $(P=0.469)$. In addition, the expression levels of these six miRNAs were significantly different between the $\mathrm{CHC}$ patients with a $\mathrm{HCV}$ genotype 1 and a non-genotype- 1 (miR-16, $P=0.001$; miR122, $P<0.001$; miR-193b, $P<0.001$; miR-199a, $P<0.001$; miR-222, $P<0.001$; and miR-324, $P=0.001$; Figure 2 ).

3.3. Expression of Six MicroRNAs Could Be Used as Biomarkers of $\mathrm{CHC}$. To further confirm whether the changes in the expression levels of these miRNAs could be used as a useful biomarker in HCV infection, we analyzed the association of the miRNA levels and clinical factors (such as ALT, AST, and liver cirrhosis) of the CHC patients. As shown in Table 4, the expression levels of the miR-16, miR-122, miR-193b, miR-199a-3p, miR-222, and miR-324-3p were significantly correlated with the changes in the ALT and AST of the $\mathrm{CHC}$ patients, but no correlation between miR-214 and these clinical factors was found (Table 4). No correlation of target miRNAs and liver cirrhosis was found in this study. Multivariate logistic regression analysis was used to verify whether the expression levels of the miRNAs acted as independent risk biomarkers for CHC (Table 5). The expression levels of the miRNAs were approximately normally distributed after log transformation. Among the variables studied were miRNA expression levels, age, gender, and BMI. The data showed that those with high expression levels of the six target miRNAs had an approximately 7.202-fold risk of CHC compared with the those with low expression levels of these miRNAs (95\% confidence interval $(\mathrm{CI})=2.014-25.752, P=0.002)$. The odds ratios of the other variables were gender $(\mathrm{OR}=0.227,95 \% \mathrm{CI}$ $=0.080-0.648$, and $P=0.006)$ and age $(\mathrm{OR}=1.182,95 \% \mathrm{CI}=$ $1.106-1.262$, and $P<0.001)$. The OR of BMI did not reach statistical significance $(\mathrm{OR}=2.090,95 \% \mathrm{CI}=0.394-11.074$, and $P=0.386$; Table 4$)$. Thus, the expression levels of six miRNAs in the PBMCs of CHC patients acted as significant risk biomarkers for the development of $\mathrm{CHC}$.

\section{Discussion}

Several studies have demonstrated a relationship between HCV and host miRNA, especially miR-122, the most abundant miRNA in the liver $[7,8,11,20]$. Previous reports 
TABLE 2: Up- and downexpressed microRNAs in PBMCs of CHC patients compared with the healthy donors.

\begin{tabular}{|c|c|c|c|c|}
\hline \multirow{3}{*}{ MicroRNAs } & \multicolumn{4}{|c|}{ Endogenous control } \\
\hline & \multicolumn{2}{|c|}{ RUN44 } & \multicolumn{2}{|c|}{ U6B } \\
\hline & $\mathrm{RQ}^{*}$ & $\log \mathrm{RQ}$ & $\mathrm{RQ}^{*}$ & $\log \mathrm{RQ}$ \\
\hline \multicolumn{5}{|c|}{ Downregulated miRNAs } \\
\hline miR-330-3p & 0.01 & -1.96 & 0 & -3.82 \\
\hline miR-371-3p & 0.02 & -1.66 & 0 & -3.53 \\
\hline miR-501-5p & 0.04 & -1.4 & 0 & -3 \\
\hline miR-636 & 0.04 & -1.37 & 0 & -3 \\
\hline miR-214 & 0.08 & -1.08 & 0 & -3 \\
\hline miR-886-3p & 0.09 & -1.07 & 0 & -3 \\
\hline miR-548c-5p & 0.11 & -0.97 & 0 & -3 \\
\hline $\operatorname{miR}-422 \mathrm{a}$ & 0.12 & -0.91 & 0 & -2.7 \\
\hline miR-886-5p & 0.24 & -0.62 & 0 & -2.25 \\
\hline miR-545 & 0.27 & -0.58 & 0 & -2.4 \\
\hline miR-326 & 0.29 & -0.54 & 0 & -2.4 \\
\hline miR-9 & 0.29 & -0.53 & 0 & -2.4 \\
\hline miR-579 & 0.38 & -0.42 & 0.01 & -2.3 \\
\hline miR-124 & 0.39 & -0.41 & 0.01 & -2.3 \\
\hline miR-627 & 0.42 & -0.38 & 0.01 & -2.22 \\
\hline $\operatorname{miR}-449 b$ & 0.5 & -0.3 & - & - \\
\hline miR-564 & 0.65 & -0.19 & 0 & -3.62 \\
\hline $\operatorname{miR}-222^{*}$ & - & - & 0 & -3.41 \\
\hline $\operatorname{miR}-15^{*}$ & - & - & 0 & -3 \\
\hline $\operatorname{miR}-16-1^{*}$ & - & - & 0 & -3 \\
\hline \multicolumn{5}{|c|}{ Upregulated miRNAs } \\
\hline miR-7-1* & 5405.77 & 3.73 & 2.01 & 0.3 \\
\hline miR-126* & 2486.35 & 3.4 & 1.3 & 0.11 \\
\hline miR-199a-3p & 814.45 & 2.91 & 10.77 & 1.03 \\
\hline miR-489 & 592.42 & 2.77 & 7.83 & 0.89 \\
\hline miR-194 & 484.64 & 2.69 & 6.41 & 0.81 \\
\hline $\operatorname{miR}-590-5 p$ & 397.96 & 2.6 & 5.26 & 0.72 \\
\hline let-7e & 311.16 & 2.49 & 4.11 & 0.61 \\
\hline miR-15b & 261.75 & 2.42 & 3.46 & 0.54 \\
\hline miR-19a & 245.95 & 2.39 & 3.25 & 0.51 \\
\hline miR-19b & 233.54 & 2.37 & 3.09 & 0.49 \\
\hline $\operatorname{miR}-376 c$ & 228.43 & 2.36 & 3.02 & 0.48 \\
\hline miR-324-5p & 224.46 & 2.35 & 2.97 & 0.47 \\
\hline $\operatorname{miR}-374 a$ & 218.5 & 2.34 & 2.89 & 0.46 \\
\hline $\operatorname{miR}-27 b$ & 193.3 & 2.29 & 2.56 & 0.41 \\
\hline miR-452 & 182.34 & 2.26 & 2.41 & 0.38 \\
\hline miR-96 & 176.66 & 2.25 & 2.34 & 0.37 \\
\hline miR-210 & 171.29 & 2.23 & 2.26 & 0.35 \\
\hline miR-139-5b & 155.02 & 2.19 & 2.05 & 0.31 \\
\hline
\end{tabular}

PBMCs: peripheral blood mononuclear cells; CHC: chronic hepatitis C.

${ }^{*}$ Real-time relative quantification $(\mathrm{RQ})$ values.

provided evidence that after knockdown miR-122 processing machinery in culture cells significantly reduced HCV viral RNA [21]. In the present study, the expression of miR-122 was not correlated with clinical parameters of $\mathrm{CHC}$, including the viral load (see Supplemental Table 1 in Supplementary Material available online at http://dx.doi.org/10.1155/2014/367157). Thus, we compared the expression levels of miR-122 in the liver tissues and PBMCs of the CHC patients. The results showed that the expression level of miR-122 was significantly lower in the PBMCs of the CHC patients than in the liver tissues (Figure 1). In addition, the level of expression of miR122 in the PBMCs of the CHC patients was significantly lower than in the healthy controls. Thus, we consider that the expression level of miR-122 in PBMCs could be used as a diagnosis marker of $\mathrm{CHC}$ but not to monitor amplification of the virus.

Changes of microRNAs in the liver have been reported in disease processes such as hepatocarcinogenesis and liver fibrosis [22-26]. However, detecting these biomarkers using tissues was difficult to apply in clinical practice to monitor 
TABLE 3: Target miRNAs expression in PBMCs of CHC patients with different HCV genotype and healthy controls.

\begin{tabular}{|c|c|c|c|c|}
\hline miRNAs & GT-1 & Non-GT-1 & Control & $P$ value \\
\hline miR-16 & $155.065(10.950)^{*}$ & $245.167(25.742)$ & $13.604(1.713)$ & $<0.001$ \\
\hline$>1.713$ & 54 & 15 & 24 & \\
\hline$\leqq 1.713$ & 12 & 5 & 24 & \\
\hline$P$ value & & & & \\
\hline miR-122 & $0.0016(0.0002)$ & $0.0000003(0)$ & $0.0031(0.0004)$ & $<0.001$ \\
\hline$>0.0004$ & 17 & 0 & 22 & \\
\hline$\leqq 0.0004$ & 54 & 20 & 26 & \\
\hline$P$ value & & & & \\
\hline miR-193b & $0.2175(0.0070)$ & $0.0068(0.00076)$ & $0.0033(0.00013)$ & $<0.001$ \\
\hline$>0.00013$ & 64 & 13 & 24 & \\
\hline$\leqq 0.00013$ & 6 & 7 & 24 & \\
\hline$P$ value & & & & \\
\hline miR-199a & $1366.614(513.816)$ & $95.7169(33.5522)$ & 479.1835 (9.4969) & $<0.001$ \\
\hline$>9.4969$ & 65 & 13 & 24 & \\
\hline$\leqq 9.4969$ & 4 & 7 & 24 & \\
\hline$P$ value & & & & \\
\hline $\operatorname{miR}-214$ & $159.1452(0.4719)$ & $677.42(0.5559)$ & $20.2853(0.2282)$ & 0.943 \\
\hline$>0.2282$ & 41 & 12 & 24 & \\
\hline$\leqq 0.2282$ & 26 & 8 & 24 & \\
\hline$P$ value & & & & \\
\hline miR-222 & $3.9360(0.4564)$ & $2.0039(0.1394)$ & $0.4898(0.0103)$ & $<0.001$ \\
\hline$>0.0103$ & 61 & 18 & 23 & \\
\hline$\leqq 0.0103$ & 5 & 2 & 24 & \\
\hline$P$ value & & & & \\
\hline miR-324 & 49.4397 (2.2015) & $21.9596(4.2781)$ & $9.4064(0.0524)$ & \\
\hline$>0.0524$ & 53 & 16 & 24 & \\
\hline$\leqq 0.0524$ & 12 & 4 & 24 & \\
\hline$P$ value & & & & \\
\hline
\end{tabular}

PBMCs: peripheral blood mononuclear cells; CHC: chronic hepatitis C.

* Data were presented by mean (medium).

the disease. Detection of miRNAs in peripheral blood, either cell-associated or cell-free, holds great potential for noninvasive and real-time molecular based monitoring of diagnosis and treatment effects in disease. Extracellular miRNAs are remarkably stable in the circulation [27-30]. Previous reports showed that some miRNAs were released from their cells of origin and could be captured in various extracellular fluids; numerous studies began investigating whether tissueand disease-specific miRNA signatures were also reflected in fluids such as blood, urine, spinal fluid, or saliva [27$29,31,32]$. As a result of cellular damage/tissue injury, such as in acute myocardial infarction (MI) [33-35], atherosclerosis [36], non-small cell lung cancer [37], neurodegenerative diseases [38-41], skin fibrosis [42], and osteoarthritis [43], miRNA expression can change in the blood. Thus, circulating miRNAs are attractive candidates for disease monitoring to serve as valuable prognostic indicators of disease progression or resolution.

There is however only limited information about their detection in blood and their correlation with histological disease severity in patients with CHC. Previous studies have elected to use miRNA from serum instead of miRNA from exosome as the candidate for diagnosing diseases [28, 44-47]. Nathwani et al. mentioned that variations in the concentration of miR-122 in serum or plasma tend to be more specific for liver diseases than ALT and AST. This is because miR122 is almost exclusively expressed in the liver, whereas ALT and AST originate from skeletal muscles and other tissues; therefore their diagnostic value is low [48]. In the present study, we also found that the expression levels of miR-16, miR-122, miR-193b, miR-199a-3p, miR-222, and miR-324-3p were significantly correlated with the changes in the ALT and AST of the CHC patients (Table 4). In addition, the present study revealed that those with high expression levels of the target miRNAs had an approximately 7.202-fold risk of CHC compared with those with low expression levels of six target miRNAs $(95 \%$ confidence interval $(\mathrm{CI})=2.014-25.752, P=$ 0.002). Thus, we consider that the expression levels of these six miRNAs in the PBMCs of CHC patients act as significant risk biomarkers for the development of $\mathrm{CHC}$.

A previous report showed that after full-length HCV was transfected into HepG2 cells, levels of miR-193b increased, whereas those of the predicted downstream target Mcl-1 gene decreased compared with a parental control [49]. Peng et al. 


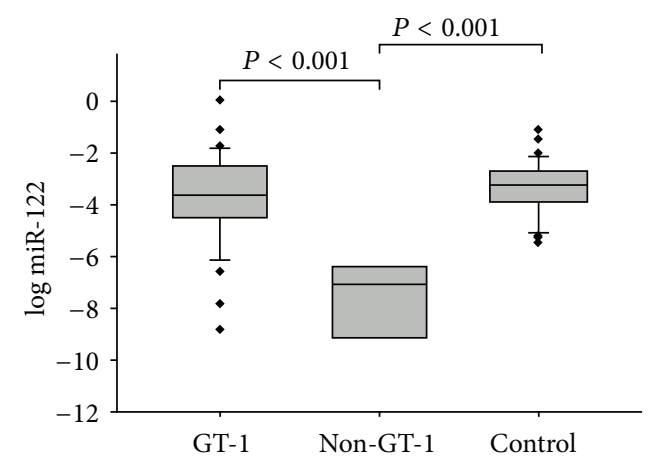

(a)

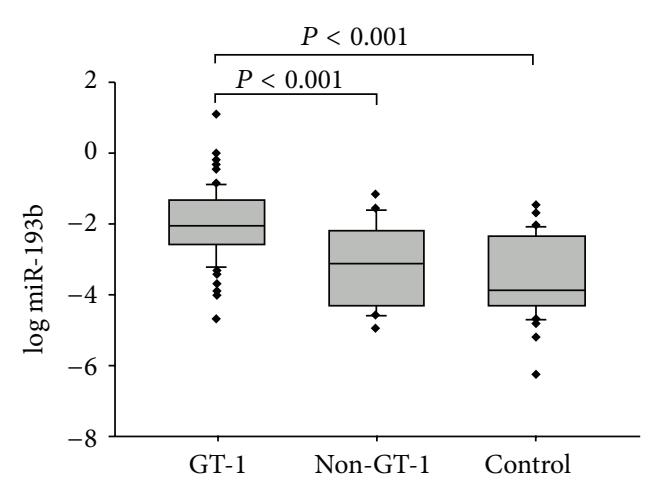

(c)

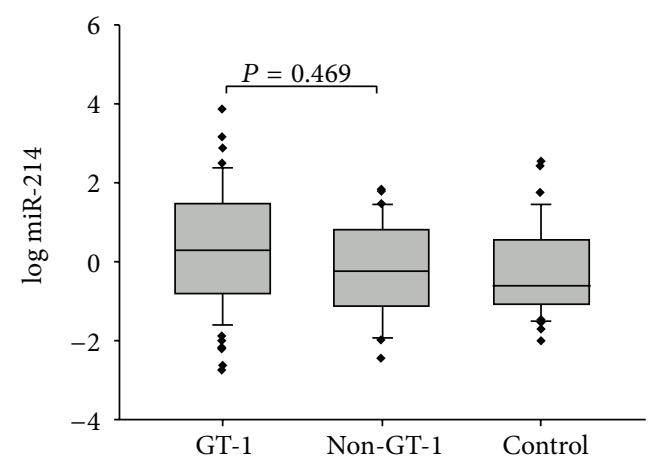

(e)

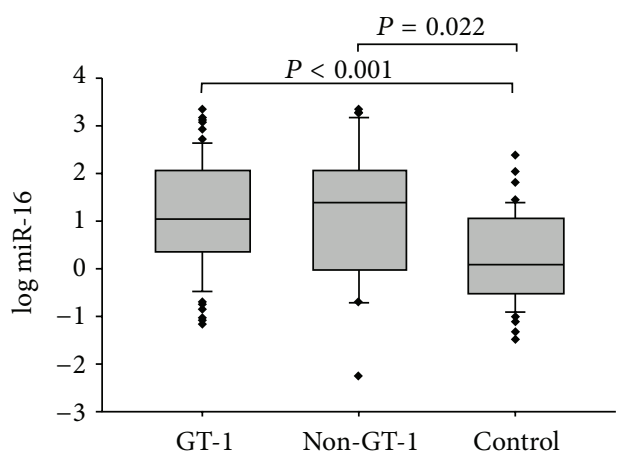

(b)

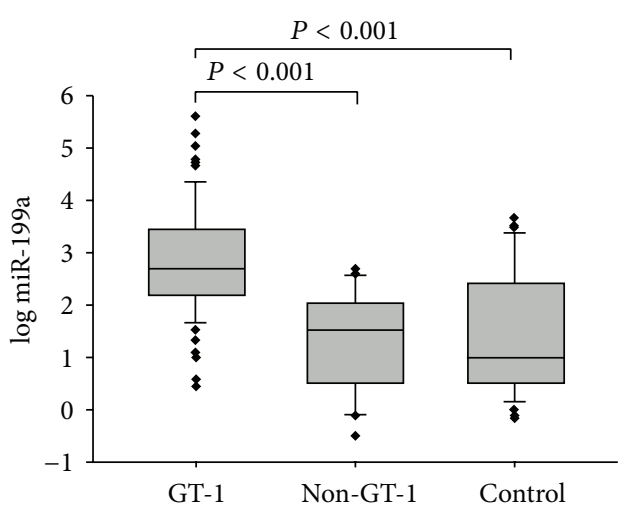

(d)

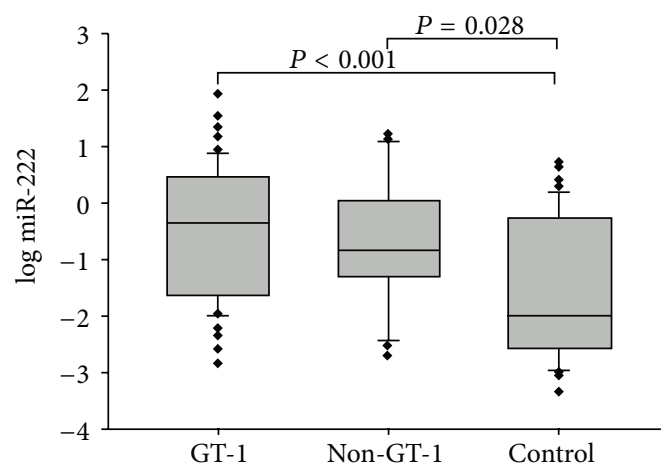

(f)

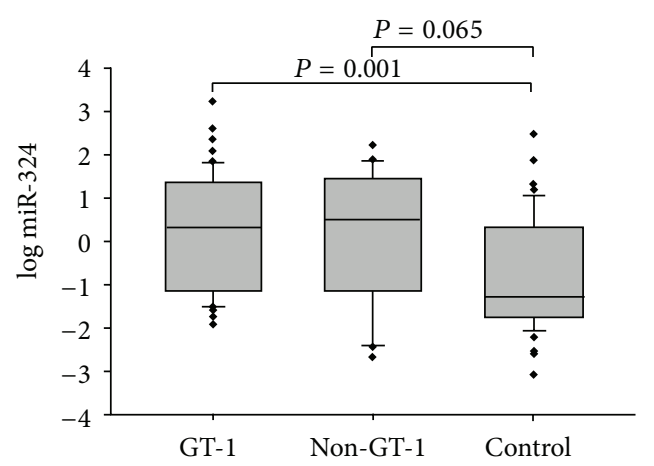

(g)

Figure 2: Target miRNAs expression in PBMCs of CHC patients with different HCV genotype and healthy control groups. 
TABLE 4: The association of the targets miRNAs expression levels and clinical factors (such as ALT, AST, and liver fibrosis) of the CHC patients.

\begin{tabular}{|c|c|c|c|c|c|c|c|}
\hline Parameters & miR-193b & miR-199a-3p & miR-122 & miR-16 & miR-214 & miR-222 & miR-324-3p \\
\hline \multicolumn{8}{|l|}{ ALT } \\
\hline$<40 \mathrm{U} / \mathrm{L}$ & $0.169 \pm 0.838$ & $777.105 \pm 2178.070$ & $0.003 \pm 0.010$ & $21.762 \pm 69.405$ & $18.220 \pm 65.577$ & $0.425 \pm 0.954$ & $7.771 \pm 39.245$ \\
\hline$\geqq 40 \mathrm{U} / \mathrm{L}$ & $0.214 \pm 1.464$ & $1113.280 \pm 497.265$ & $0.001 \pm 0.002$ & $212.344 \pm 504.989$ & $54.410 \pm 200.481$ & $3.425 \pm 11.770$ & $26.781 \pm 67.231$ \\
\hline$P$ value & 0.001 & 0.009 & $<0.0001$ & $<0.0001$ & 0.235 & $<0.0001$ & 0.001 \\
\hline \multicolumn{8}{|l|}{ AST } \\
\hline$<40 \mathrm{U} / \mathrm{L}$ & $0.027 \pm 0.005$ & $426.223 \pm 1115.870$ & $0.003 \pm 0.012$ & $13.217 \pm 40.283$ & $17.412 \pm 64.851$ & $0.496 \pm 1.135$ & $11.157 \pm 48.112$ \\
\hline$\geqq 40 \mathrm{U} / \mathrm{L}$ & $0.174 \pm 1.297$ & $882.580 \pm 436.044$ & $0.001 \pm 0.003$ & $168.314 \pm 445.087$ & $45.757 \pm 177.139$ & $2.631 \pm 10.244$ & $20.214 \pm 58.935$ \\
\hline$P$ value & $<0.0001$ & $<0.0001$ & $<0.0001$ & $<0.0001$ & 0.760 & $<0.0001$ & 0.001 \\
\hline \multicolumn{8}{|c|}{ Liver cirrhosis } \\
\hline No & $0.228 \pm 1.410$ & $964.452 \pm 454.544$ & $0.017 \pm 0.122$ & $197.502 \pm 490.549$ & $150.749 \pm 891.345$ & $2.529 \pm 5.821$ & $45.846 \pm 209.718$ \\
\hline Yes & $0.012 \pm 0.029$ & $1340.147 \pm 440.160$ & $0.001 \pm 0.003$ & $109.524 \pm 243.190$ & $40.417 \pm 79.656$ & $6.452 \pm 19.406$ & $34.221 \pm 86.321$ \\
\hline$P$ value & 0.221 & 0.800 & 0.866 & 0.996 & 0.054 & 0.584 & 0.508 \\
\hline
\end{tabular}

AST: aspartate aminotransferase; ALT: alanine aminotransferase.

TABLE 5: Multivariate logistic regression analysis of the risk of $\mathrm{CHC}$.

\begin{tabular}{lccc}
\hline Parameters & Favorable/unfavorable & OR & $95 \%$ CI \\
\hline Gender & Female/male & 0.227 & $0.080-0.648$ \\
Age & Per year & 1.182 & $1.1062013 ; 1.262$ \\
BMI & $\geqq 27 /<27$ & 2.090 & 0.006 \\
Six target miRNAs & High/low & 7.202 & $2.014-25.727$ \\
\hline
\end{tabular}

Six target miRNAs were including miR-16, miR-122, miR-193b, miR-199a-3p, miR-222, and miR-324-3p.

In CHC patients' PBMC, the expression levels of miR-16, miR-122, miR-193b, miR-199a-3p, miR-222, and miR-324-3p were increased and miR-122 expression was decreased compared with healthy controls.

(2009) carried out a computational study of HCV associated miRNA-mRNA regulatory modes in human livers. They found that miR-122, miR-320, and miR-191 were downregulated, whereas miR-215, miR-16, miR-26, miR-130, miR-199, and miR-155 were upregulated [11]. Murakami et al. (2009) demonstrated that miR-199a* can control HCV viral replication [9]. The current study showed that levels of miR-193b and miR-199a-3p were significantly higher in the PBLs of HCV patients than in the control group. In addition, we found that those with high levels of expression of miR-193b and miR199a-3p had an approximately 6.545-fold risk of CHC compared with those with low levels of expression of miR-193b and miR-199a-3p (95\% CI = 2.180-19.651, $P=0.001$; Supplemental Table 2). Thus, we suggest that the expression level of miRNAs may serve as a useful biomarker for HCV infection.

In conclusion, we found specific expression patterns of miRNAs in CHC patients compared with healthy controls, suggesting the potential contribution of miRNA activities to the pathobiology of HCV infection. Changes in the levels of miRNAs in PBMCs in CHC may act as significant diagnosis biomarkers for $\mathrm{CHC}$.

\section{Conflict of Interests}

The authors do not have any commercial or other associations that might pose a conflict of interests.

\section{Authors' Contribution}

Ya-Wen Cheng and Chun-Che Lin designed the study and wrote the paper; Chiu-Chun Chang and Wan-Ling Hsieh designed the experiments and prepared the figures; ChunChe Lin collected the CHC and control samples; Chiu-Chun Chang and Wan-Ling Hsieh analyzed data. All authors gave final approval for the paper to be submitted for publication. Chiu-Chun Chang and Chun-Che Lin equally contributed to this study.

\section{Acknowledgments}

This study was supported by grant of the National Science Council (NSC 100-2314-B-040-012) and Chung Shan Medical University Hospital (CSH-2012-C-016).

\section{References}

[1] J. A. Mengshol, L. Golden-Mason, and H. R. Rosen, "Mechanisms of disease: HCV-induced liver injury," Nature Clinical Practice Gastroenterology \& Hepatology, vol. 4, no. 110, pp. 622634, 2007.

[2] R.-X. Shao, Y. Hoshida, M. Otsuka et al., "Hepatic gene expression profiles associated with fibrosis progression and hepatocarcinogenesis in hepatitis C patients," World Journal of Gastroenterology, vol. 11, no. 13, pp. 1995-1999, 2005.

[3] D. P. Bartel, "MicroRNAs: genomics, biogenesis, mechanism, and function," Cell, vol. 116, no. 2, pp. 281-297, 2004.

[4] M. Lagos-Quintana, R. Rauhut, A. Yalcin, J. Meyer, W. Lendeckel, and T. Tuschl, "Identification of tissue-specific MicroRNAs from mouse," Current Biology, vol. 12, no. 9, pp. 735-739, 2002.

[5] B. Ason, D. K. Darnell, B. Wittbrodt et al., "Differences in vertebrate microRNA expression," Proceedings of the National 
Academy of Sciences of the United States of America, vol. 103, no. 39, pp. 14385-14389, 2006.

[6] P. S. Mitchell, R. K. Parkin, E. M. Kroh et al., "Circulating microRNAs as stable blood-based markers for cancer detection," Proceedings of the National Academy of Sciences of the United States of America, vol. 105, no. 30, pp. 10513-10518, 2008.

[7] K. Wang, S. Zhang, B. Marzolf et al., "Circulating microRNAs, potential biomarkers for drug-induced liver injury," Proceedings of the National Academy of Sciences of the United States of America, vol. 106, no. 11, pp. 4402-4407, 2009.

[8] C. L. Jopling, M. Yi, A. M. Lancaster, S. M. Lemon, and P. Sarnow, "Molecular biology: modulation of hepatitis $\mathrm{C}$ virus RNA abundance by a liver-specific MicroRNA," Science, vol. 309, no. 5740, pp. 1577-1581, 2005.

[9] Y. Murakami, H. H. Aly, A. Tajima, I. Inoue, and K. Shimotohno, "Regulation of the hepatitis $\mathrm{C}$ virus genome replication by miR199a," Journal of Hepatology, vol. 50, no. 3, pp. 453-460, 2009.

[10] S. Bala, M. Marcos, and G. Szabo, "Emerging role of microRNAs in liver diseases," World Journal of Gastroenterology, vol. 15, no. 45, pp. 5633-5640, 2009.

[11] X. Peng, Y. Li, K.-A. Walters et al., "Computational identification of hepatitis $C$ virus associated microRNA-mRNA regulatory modules in human livers," BMC Genomics, vol. 10, article 373, 2009.

[12] A. J. Atkinson Jr., W. A. Colburn, V. G. DeGruttola et al., "Biomarkers and surrogate endpoints: Preferred definitions and conceptual framework. Biomarkers Definitions Working Group," Clinical Pharmacology \& Therapeutics, vol. 69, no. 3, pp. 89-95, 2001.

[13] J. Woodcock, A Framework for Biomarker and Surrogate Endpoint Use in Drug Development, http://www.fda.gov/ohrms/ dockets/ac/04/slides/2004-4079S2_03_Woodcock.ppt.

[14] National Cancer Institute, Dictionary of Cancer Terms, http://www.cancer.gov/dictionary/?searchTxt=biomarker.

[15] M. J. Koziel, "The role of immune responses in the pathogenesis of hepatitis C virus infection," Journal of Viral Hepatitis, vol. 4, supplement 2, pp. 31-41, 1997.

[16] S. Bala, Y. Tilahun, O. Taha et al., "Increased microRNA-155 expression in the serum and peripheral monocytes in chronic HCV infection," Journal of Translational Medicine, vol. 10, no. 1, article 151, 2012.

[17] R. S. Tacke, A. Tosello-Trampont, V. Nguyen, D. W. Mullins, and Y.S. Hahn, "Extracellular hepatitis $\mathrm{C}$ virus core protein activates STAT3 in human monocytes/macrophages/dendritic cells via an IL-6 autocrine pathway," The Journal of Biological Chemistry, vol. 286, no. 12, pp. 10847-10855, 2011.

[18] M. Grek, A. Piekarska, J. Bartkowiak et al., "Coordinated increase of miRNA-155 and miRNA-196b expression correlates with the detection of the antigenomic strand of hepatitis $C$ virus in peripheral blood mononuclear cells," International Journal of Molecular Medicine, vol. 28, no. 5, pp. 875-880, 2011.

[19] D. Otaegui, S. E. Baranzini, R. Armañanzas et al., "Differential micro RNA expression in PBMC from multiple sclerosis patients," PLoS ONE, vol. 4, no. 7, Article ID e6309, 2009.

[20] G. Szabo and S. Bala, "MicroRNAs in liver disease," Nature Reviews Gastroenterology \& Hepatology, vol. 10, no. 11, pp. 542552, 2013.

[21] G. Randall, M. Panis, J. D. Cooper et al., "Cellular cofactors affecting hepatitis $\mathrm{C}$ virus infection and replication," Proceedings of the National Academy of Sciences of the United States of America, vol. 104, no. 31, pp. 12884-12889, 2007.
[22] Y. Murakami, A. Tamori, S. Itami et al., "The expression level of miR-18b in hepatocellular carcinoma is associated with the grade of malignancy and prognosis," BMC Cancer, vol. 13, article 99, 2013.

[23] Y. Zhang, S. Takahashi, A. Tasaka, T. Yoshima, H. Ochi, and K. Chayama, "Involvement of microRNA-224 in cell proliferation, migration, invasion, and anti-apoptosis in hepatocellular carcinoma," Journal of Gastroenterology and Hepatology, vol. 28, no. 3, pp. 565-575, 2013.

[24] G. Lendvai, A. Kiss, I. Kovalszky, and Z. Schaff, "MicroRNAs in hepatocarcinogenesis," Orvosi Hetilap, vol. 153, no. 25, pp. 978 989, 2012.

[25] A. Karakatsanis, I. Papaconstantinou, M. Gazouli, A. Lyberopoulou, G. Polymeneas, and D. Voros, "Expression of microRNAs, miR-21, miR-31, miR-122, miR-145, miR-146a, miR-200c, miR-221, miR-222, and miR-223 in patients with hepatocellular carcinoma or intrahepatic cholangiocarcinoma and its prognostic significance," Molecular Carcinogenesis, vol. 52, no. 4, pp. 297-303, 2013.

[26] I. Papaconstantinou, A. Karakatsanis, M. Gazouli, G. Polymeneas, and D. Voros, "The role of microRNAs in liver cancer," European Journal of Gastroenterology and Hepatology, vol. 24, no. 3, pp. 223-228, 2012.

[27] X. Chen, Y. Ba, L. Ma et al., "Characterization of microRNAs in serum: a novel class of biomarkers for diagnosis of cancer and other diseases," Cell Research, vol. 18, no. 10, pp. 997-1006, 2008.

[28] M. P. Hunter, N. Ismail, X. Zhang et al., "Detection of microRNA expression in human peripheral blood microvesicles," PLoS ONE, vol. 3, no. 11, Article ID e3694, 2008.

[29] P. S. Mitchell, R. K. Parkin, E. M. Kroh et al., "Circulating microRNAs as stable blood based markers for cancer detection," Proceedings of the National Academy of Sciences of the United States of America, vol. 105, no. 30, pp. 10513-10518, 2008.

[30] S. Gilad, E. Meiri, Y. Yogev et al., "Serum microRNAs are promising novel biomarkers," PLoS ONE, vol. 3, no. 9, Article ID e3148, 2008.

[31] M. A. Cortez and G. A. Calin, "MicroRNA identification in plasma and serum: a new tool to diagnose and monitor diseases," Expert Opinion on Biological Therapy, vol. 9, no. 6, pp. 703-711, 2009.

[32] J. A. Weber, D. H. Baxter, S. Zhang et al., "The microRNA spectrum in 12 body fluids," Clinical Chemistry, vol. 56, no. 11, pp. 1733-1741, 2010.

[33] T. Adachi, M. Nakanishi, Y. Otsuka et al., "Plasma microRNA 499 as a biomarker of acute myocardial infarction," Clinical Chemistry, vol. 56, no. 7, pp. 1183-1185, 2010.

[34] J. Ai, R. Zhang, Y. Li et al., "Circulating microRNA-1 as a potential novel biomarker for acute myocardial infarction," Biochemical and Biophysical Research Communications, vol. 391, no. 1, pp. 73-77, 2010.

[35] C. Widera, S. K. Gupta, J. M. Lorenzen et al., "Diagnostic and prognostic impact of six circulating microRNAs in acute coronary syndrome," Journal of Molecular and Cellular Cardiology, vol. 51, no. 5, pp. 872-875, 2011.

[36] R. Menghini, V. Casagrande, and M. Federici, "MicroRNAs in endothelial senescence and atherosclerosis," Journal of Cardiovascular Translational Research, vol. 6, no. 6, pp. 924-930, 2013.

[37] Y. Huang, Q. Hu, Z. Deng, Y. Hang, J. Wang, and K. Wang, "Micrornas in body fluids as biomarkers for non-small cell lung cancer: a systematic review," Technology in Cancer Research \& Treatment, vol. 13, no. 3, pp. 277-287, 2014. 
[38] L. Cheng, C. Y. J. Quek, X. Sun, S. A. Bellingham, and A. F. Hill, "The detection of microRNA associated with Alzheimer's disease in biological fluids using next-generation sequencing technologies," Frontiers in Genetics, vol. 4, article 150, 2013.

[39] V. Dorval, P. T. Nelson, and S. S. Hébert, "Circulating microRNAs in Alzheimer's disease: the search for novel biomarkers," Frontiers in Molecular Neuroscience, vol. 6, article 24, 2013.

[40] X.-F. Jin, N. Wu, L. Wang, and J. Li, "Circulating microRNAs: a novel class of potential biomarkers for diagnosing and prognosing central nervous system diseases," Cellular and Molecular Neurobiology, vol. 33, no. 5, pp. 601-613, 2013.

[41] K. S. Sheinerman and S. R. Umansky, "Circulating cell-free microRNA as biomarkers for screening, diagnosis, and monitoring of neurodegenerative diseases and other neurologic pathologies," Frontiers in Cellular Neuroscience, vol. 7, Article ID 150, 2013.

[42] O. Babalola, A. Mamalis, H. Lev-Tov, and J. Jagdeo, “The role of microRNAs in skin fibrosis," Archives of Dermatological Research, vol. 305, no. 9, pp. 763-776, 2013.

[43] A. Gonzalez, "Osteoarthritis year 2013 in review: genetics and genomics," Osteoarthritis and Cartilage, vol. 21, no. 10, pp. 14431451, 2013.

[44] X. Chen, Y. Ba, L. Ma et al., "Characterization of microRNAs in serum: a novel class of biomarkers for diagnosis of cancer and other diseases," Cell Research, vol. 18, no. 10, pp. 997-1006, 2008.

[45] S. Cermelli, A. Ruggieri, J. A. Marrero, G. N. Ioannou, and L. Beretta, "Circulating microRNAs in patients with chronic hepatitis C and non-alcoholic fatty liver disease," PLoS ONE, vol. 6, no. 8, Article ID e23937, 2011.

[46] F. Ji, B. Yang, X. Peng, H. Ding, H. You, and P. Tien, "Circulating microRNAs in hepatitis B virus-infected patients," Journal of Viral Hepatitis, vol. 18, no. 7, pp. e242-e251, 2011.

[47] R. T. Marquez, S. Bandyopadhyay, E. B. Wendlandt et al., "Correlation between microRNA expression levels and clinical parameters associated with chronic hepatitis $\mathrm{C}$ viral infection in humans," Laboratory Investigation, vol. 90, no. 12, pp. 1727-1736, 2010.

[48] R. A. Nathwani, S. Pais, T. B. Reynolds, and N. Kaplowitz, "Serum alanine aminotransferase in skeletal muscle diseases," Hepatology, vol. 41, no. 2, pp. 380-382, 2005.

[49] C. Braconi, N. Valeri, P. Gasparini et al., "Hepatitis C virus proteins modulate microRNA expression and chemosensitivity in malignant hepatocytes," Clinical Cancer Research, vol. 16, no. 3, pp. 957-966, 2010. 


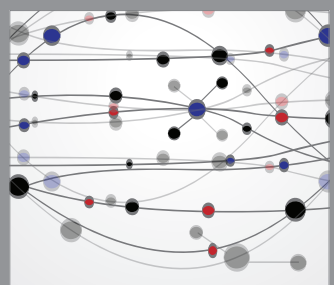

The Scientific World Journal
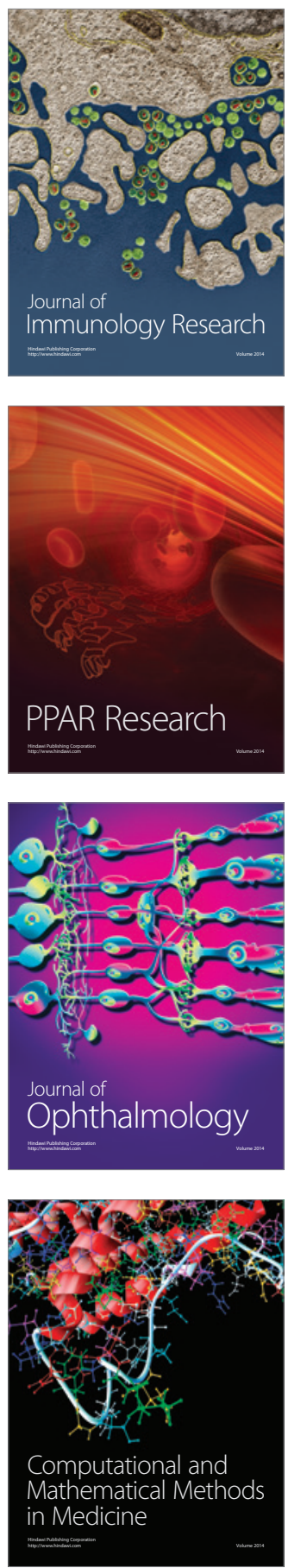

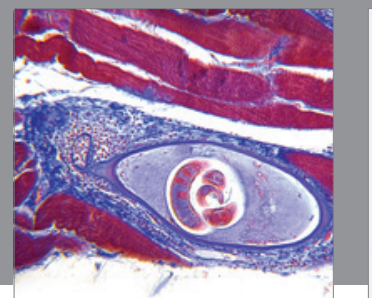

Gastroenterology

Research and Practice
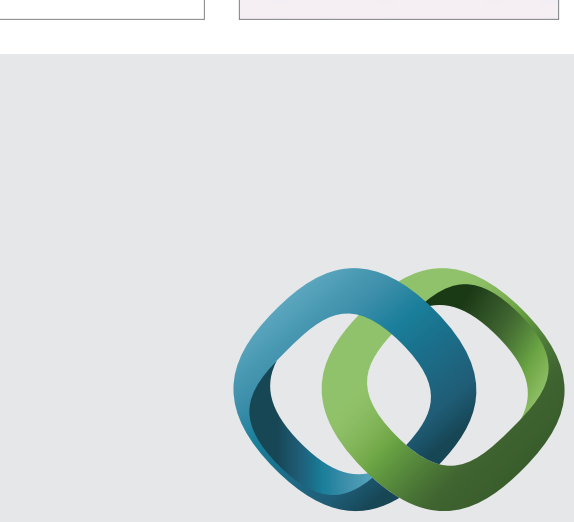

\section{Hindawi}

Submit your manuscripts at

http://www.hindawi.com
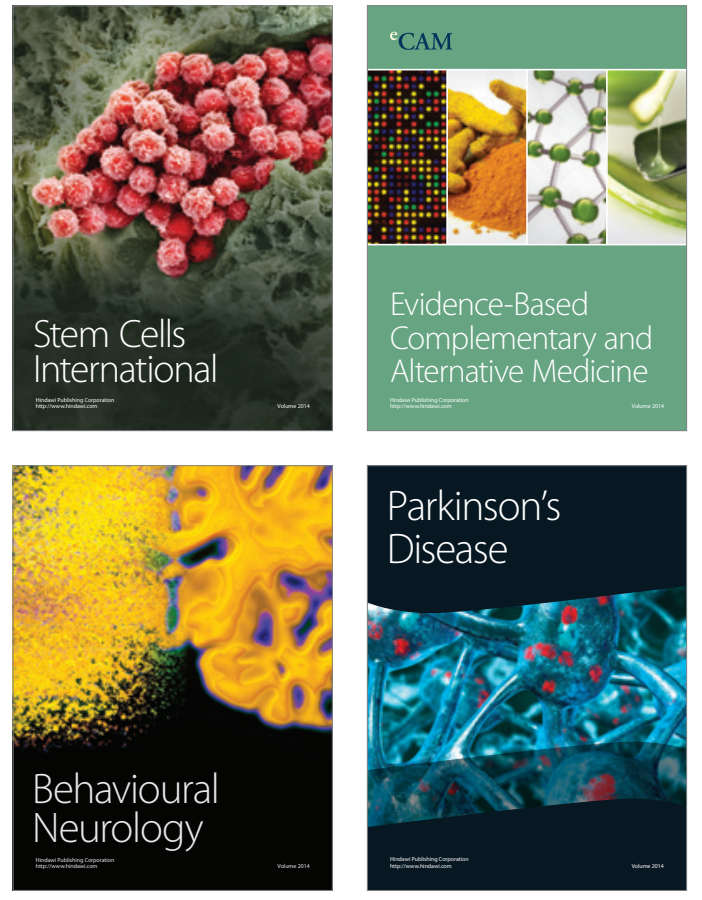
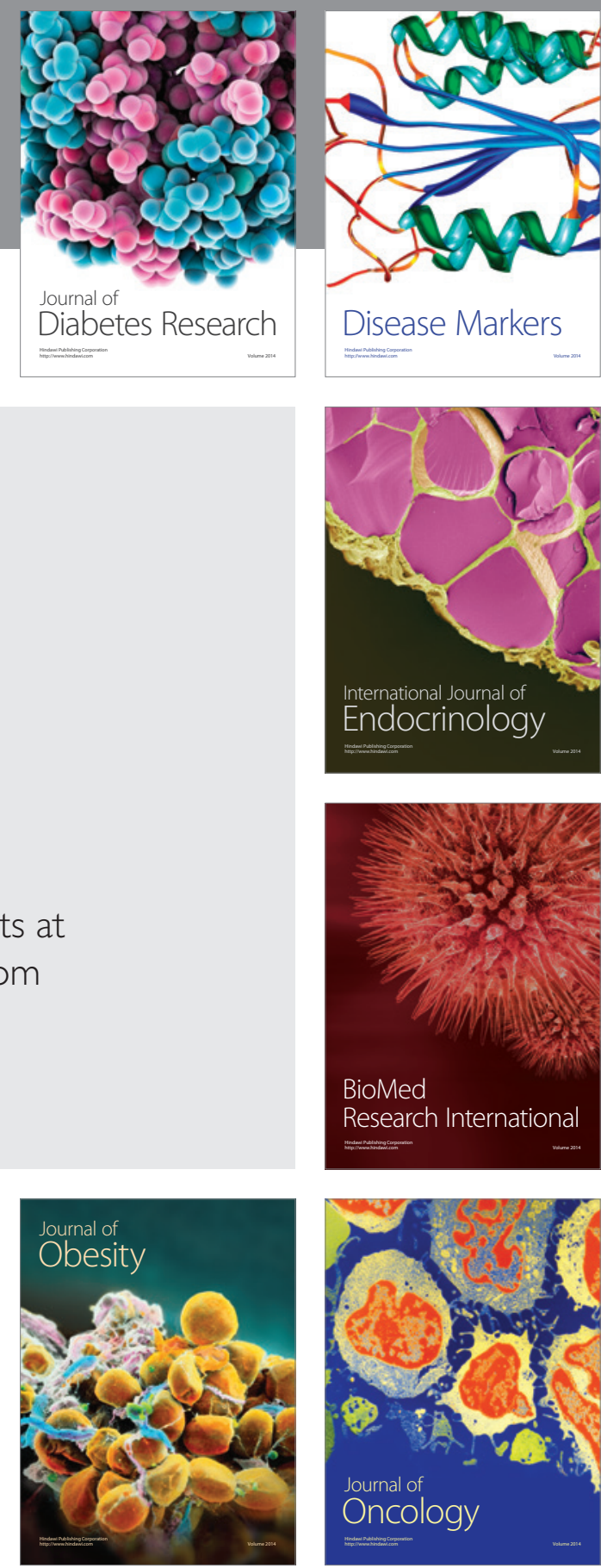

Disease Markers
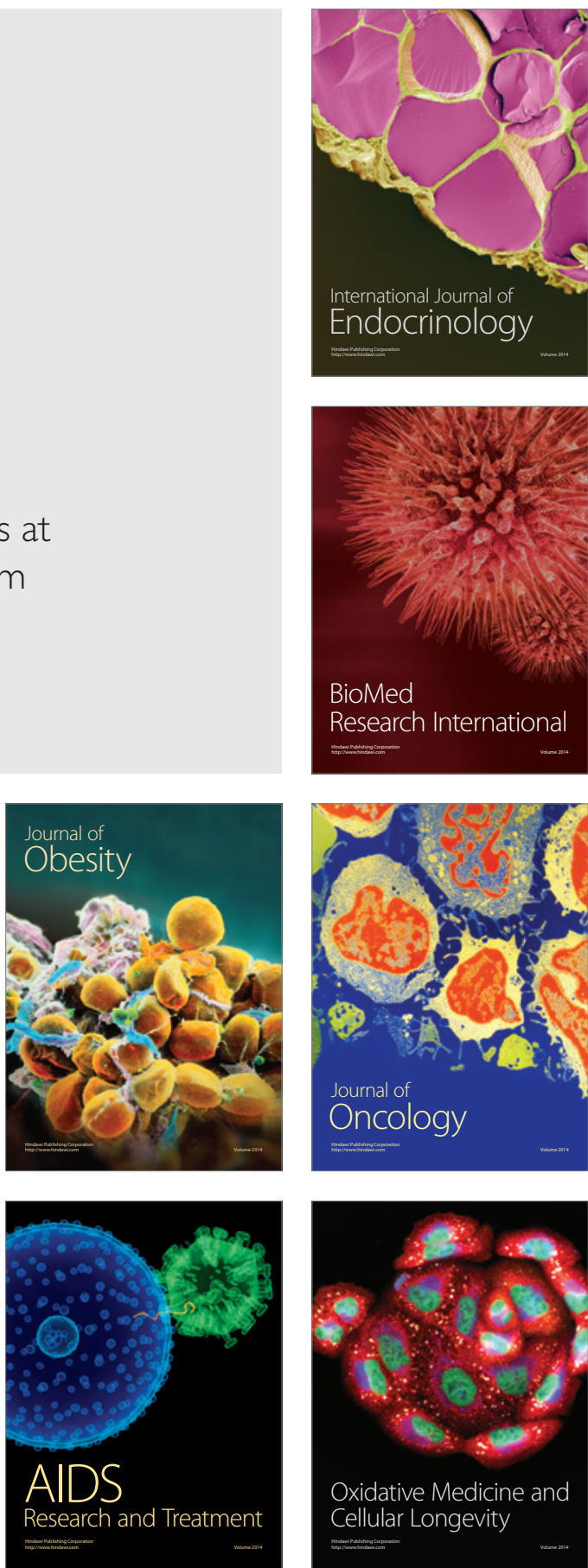